
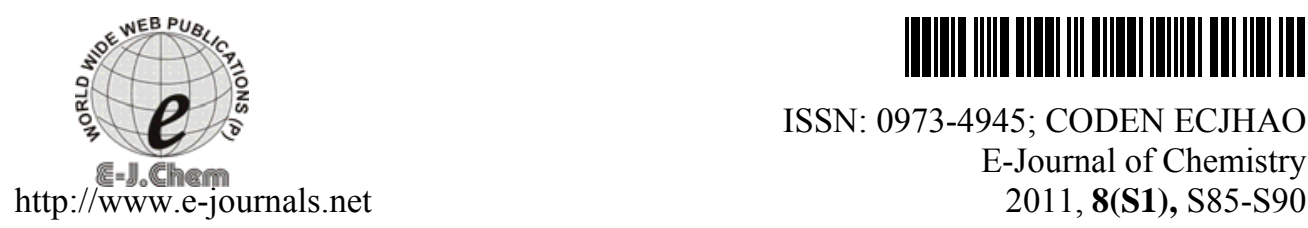

ISSN: 0973-4945; CODEN ECJHAO

E-Journal of Chemistry

2011, 8(S1), S85-S90

\title{
Synthesis, Characterization of Some Novel Benzimidazole Derivatives of 1-Bromo-2, 4-dinitrobenzene and Their Antifungal Activities
}

\author{
ABBAS AHMADI* and BABAK NAHRI-NIKNAFS \\ Department of Chemistry, Faculty of Science \\ Islamic Azad University, Karaj baranch, Karaj, Iran \\ niknafs22@yahoo.com
}

Received 16 February 2011; Accepted 16 April 2011

\begin{abstract}
Six novel benzimidazole derivatives, 5-nitro-2-phenyl -1-ethyl benzimidazol (5), 2- ( $p$-bromophenyl)- 5-nitro- 1-ethyl benzimidazol (6), 2- (p-bromophenyl-5-nitro-1-cyclopentyl benzimidazol (7), 2- (p-bromophenyl) -5-nitro-1-cyclopentyl benzimidazol (8), 5-amino-2-( $p$-bromophenyl)-1ethylbenzimidazol (9) and 5-amino-2-(p-bromophenyl)-1-cyclopentyl benzimidazol (10) were synthesized. The structures of all the synthesized compounds were elucidated by using elemental analysis and different spectroscopic techniques (IR, NMR and mass spectroscopy). Some of these compounds showed potential antifungal activities. The biological activity of these compounds as fungicides was tested against Candida albicans, patient isolate Candida glabrata and Candida krusei. The biological activity of four compounds was found to be comparable to that of the commercially available fungicides with a minimum inhibitory concentration of $12.5 \mu \mathrm{g} / \mathrm{mL}$.
\end{abstract}

Keywords: Benzimidazole, 1-Bromo-2, 4-Dinitrobenzene, Antifungal activity, Fungicides

\section{Introduction}

Benzimidazole nucleus is an important heterocyclic ring, a wide variety of benzimidazole derivatives are known for their chemotherapeutic importance and antimicrobial activities ${ }^{1-6}$, especially antifungal activity ${ }^{7-9}$ anti-inflammatory ${ }^{10}$ and antioxidant ${ }^{11-15}$. In this context, It has been found that benzimidazole derivatives to retard especial type of fungus that attack certain class of patients such as cancer chemotherapy and HIV patients. In particular, candidiasies is the fungal infection most that is frequently associated with HIV-positive patients ${ }^{16-17}$. Benzimidazole derivatives were found to retard cryptococcosis growth, which is the main cause of morbidity in AIDS patients. benzimidazole fungicides are systemic 
pesticides widely used in agriculture for pre- and post-harvest treatment for control of a wide range of fungi ${ }^{18-20}$. The limited number of available antifungal compounds urges to synthesis new compounds with a potential use as fungicides, in particular, those attack people suppressed immune system e.g. candidiasies is the fungus infection that is most frequently associated with HIV-positive patients. We report in this work, synthesis of a six benzimidazoles compounds (Figure 1) of the title structure type containing the above mentioned moieties for evaluation of their antifungal activity. Four of the six investigated compounds showed antifungal properties.

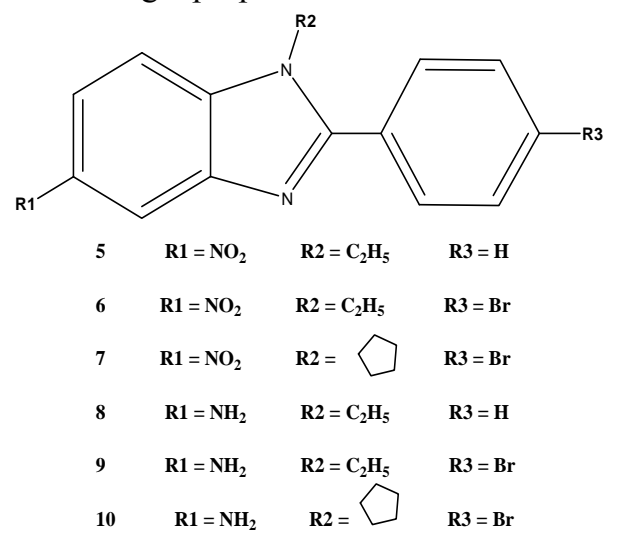

Figure 1. Chemical structures of chemical compound synthesized

\section{Experimental}

All the chemicals and solvents were obtained from E-Merck (Darmstadt, Germany) and were used without further purification. All melting points are uncorrected and were taken with an electrothermal melting point apparatus (Electrothermal Eng. Ltd, Essex, UK). IR spectra were determinate in $\mathrm{KBr}$ on a Shimadzu Dr-8031 instrument. The ${ }^{1} \mathrm{H}$ NMR spectra of the synthesized compounds were measured in DMSO- $\mathrm{d}_{6}$ or $\mathrm{CDCl}_{3}$ solution and TMS as the internal standard using a Varian Mercury $400,400 \mathrm{MHz}$ instrument. All chemical shifts were reported as $\delta$ (ppm) values. The mass spectra were recorded on a LCQ ion trap mass spectrometer (Thermo Fisher. San Jose.CA, USA), equipped with an EI source. Elemental analyses were carried out using a Perkin-Elmer, CHN elemental analyzer model 2400 and were within $\pm 0.4 \%$ of the theoretical values.

\section{General procedure for the preparation of the compounds (5-7)}

To a mixture of the appropriate aldehyde derivative $(1.5 \mathrm{mmol})$ in $5 \mathrm{~mL}$ of EtOH, then was added a solution of 0.01 mole of $\mathrm{Na}_{2} \mathrm{~S}_{2} \mathrm{O}_{5}$ in $5 \mathrm{~mL}$ of water in portions to the cooled ethanolic solution. The precipitate formed was filtered off and dried. A total of $1.2 \mathrm{mmol}$ of this precipitate and $1.2 \mathrm{mmol}$ of compound 3 or 4 in $5 \mathrm{~mL}$ of DMF were heated under reflux for $8 \mathrm{~h}$, then it was concentrated. At the end of this period the reaction mixture was cooled and poured into water and the resulting solid was collected, washed with water. The precipitate re-crystallized from ethanol-water mixture ${ }^{21}$.

\section{5-Nitro-2-phenyl -1-ethyl benzimidazol (5)}

Yield 65\%, m. p: $123-124{ }^{\circ} \mathrm{C}$; IR $\left(\mathrm{KBr}, \mathrm{cm}^{-1}\right): 2995(\mathrm{CH}), 1645(\mathrm{~N}=\mathrm{C}), 1292.4(\mathrm{C}-\mathrm{N}$ stretching), 892.1 (C-C bonding aromatic); ${ }^{1} \mathrm{H}$ NMR (DMSO-d $\left.{ }_{6}, \delta \mathrm{ppm}\right): 0.73$ (t, $3 \mathrm{H}, \mathrm{CH}_{3}$ ), 
1.66-1.71 (m, $\left.2 \mathrm{H}, \mathrm{CH}_{2}\right), 2.5\left(3 \mathrm{H}, \mathrm{s}, \mathrm{CH}_{3}\right.$ at $\mathrm{C}-2$ of benzimidazole $), 4.37\left(\mathrm{t}, 2 \mathrm{H}, \mathrm{CH}_{2}\right)$, 7.21-7.59 (4H, m, Ar-bbenzimidazole), 7.62-7.65 (m, 3H, H-3', $\left.4^{\prime}, 5^{\prime}\right), 7.81-7.98(\mathrm{~m}, 2 \mathrm{H}$, H-2',6'), 7.96 (d, 1H, Jo= 8.8 Hz, H-7), 8.24 (dd, 1H, Jo =8.8 Hz, Jm=2 Hz, H-6), 8.59 $(\mathrm{d}, 1 \mathrm{H}, \mathrm{Jm}=2 \mathrm{~Hz}, \mathrm{H}-4), 12.5\left(1 \mathrm{H}, \mathrm{s},-\mathrm{NH}-\right.$ Benzimidazole). Anal. Calcd. For $\mathrm{C}_{15} \mathrm{H}_{13} \mathrm{~N}_{3} \mathrm{O}_{2}: \mathrm{C}, 67.42$; H, 4.87; N, 15.73. Found: C, 67.31; H, 4.81; N, 15.62; Mass spectra, $\mathrm{m} / \mathrm{z}=267.20$ (100\%).

\section{2- (p-Bromophenyl)-5-nitro- 1-ethyl benzimidazol (6)}

Yield 62\%, m. p: $157-158{ }^{\circ} \mathrm{C}$; IR $\left(\mathrm{KBr}, \mathrm{cm}^{-1}\right): 2995(\mathrm{CH}), 1655(\mathrm{~N}=\mathrm{C}), 1291(\mathrm{C}-\mathrm{N}$ stretching), 895 (C-C bonding aromatic), $667(\mathrm{C}-\mathrm{Br}) ;{ }^{1} \mathrm{HNMR}$ (DMSO-d $\left.\mathrm{d}_{6}, \delta \mathrm{ppm}\right): 0.73$ $\left(\mathrm{t}, 3 \mathrm{H}, \mathrm{CH}_{3}\right), 1.65-1.71\left(\mathrm{~m}, 2 \mathrm{H}, \mathrm{CH}_{2}\right), 2.5\left(3 \mathrm{H}, \mathrm{s}, \mathrm{CH}_{3}\right.$ at $\mathrm{C}-2$ of benzimidazole $), 4.35(\mathrm{t}, 2 \mathrm{H}$, $\mathrm{CH}_{2}$ ), 7.22-7.65 (4H, m, Ar-Bbenzimidazole), 7.45-7.49 (m, 2H, H-3',5'), 7.88-7.91 (m, 2H, H-2',6'), 7.96 (d, 1H, Jo= $8.8 \mathrm{~Hz}, \mathrm{H}-7), 8.22$ (d, 1H, Jo= $8.8 \mathrm{~Hz}, \mathrm{H}-6), 8.58$ (s, 1H, H-4). $12.8(1 \mathrm{H}, \mathrm{s},-\mathrm{NH}-\mathrm{Benzimidazole})$. Anal. Calcd. For $\mathrm{C}_{15} \mathrm{H}_{12} \mathrm{BrN}_{3} \mathrm{O}_{2}: \mathrm{C}, 52.04 ; \mathrm{H}, 3.46 ; \mathrm{N}$, 12.13, Found: C, 52.00; H, 3.40; N, 12.03; Mass spectra, m/z=346.10 (100\%).

\section{2- (p-Bromophenyl)-5-nitro-1- cyclopentyl benzimidazol (7)}

Yield 85\%, m. p.: $172-173{ }^{\circ} \mathrm{C}$; IR $\left(\mathrm{KBr}, \mathrm{cm}^{-1}\right): 2923(\mathrm{CH}), 1624(\mathrm{~N}=\mathrm{C}), 1291(\mathrm{C}-\mathrm{N}$ stretching), 901 (C-C bonding aromatic), 681 (C-Br); ${ }^{1} \mathrm{HNMR}$ (DMSO-d $\left.{ }_{6}, \delta \mathrm{ppm}\right): 1.68$ $2.16\left(\mathrm{~m}, 8 \mathrm{H}, \mathrm{CH}_{2}\right), 2.5\left(3 \mathrm{H}, \mathrm{s}, \mathrm{CH}_{3}\right.$ at $\mathrm{C}-2$ of benzimidazole $), 4.85-4.89(\mathrm{~m}, 1 \mathrm{H}, \mathrm{CH}), 7.20-$ $7.60(4 \mathrm{H}, \mathrm{m}$, Ar-bbenzimidazole) 7.45-7.49 (m, 2H, H-3',5'), 7.78-7.82 (m, 2H, H-2',6'), $7.89(\mathrm{~d}, 1 \mathrm{H}, \mathrm{Jo}=9.2 \mathrm{~Hz}, \mathrm{H}-7), 8.17(\mathrm{dd}, 1 \mathrm{H}, \mathrm{Jo}=9.2 \mathrm{~Hz}, \mathrm{Jm}=2 \mathrm{~Hz}, \mathrm{H}-6), 8.58(\mathrm{~d}, 1 \mathrm{H}, \mathrm{J}$ $\mathrm{m}=1.6 \mathrm{~Hz}, \mathrm{H}-4), 12.9\left(1 \mathrm{H}, \mathrm{s},-\mathrm{NH}-\right.$ benzimidazole) Anal. Calcd. For $\mathrm{C}_{18} \mathrm{H}_{16} \mathrm{BrN}_{3} \mathrm{O}_{2}: \mathrm{C}, 55.97$; H, 4.14; N, 10.87, Found: C, 55.90; H, 4.11; N, 10.81; Mass spectra, m/z=386.20 (100\%).

\section{General procedure for the preparation of the compounds (8-10)}

Mixture of 5-nitrobenzimidazole derivatives 5-7 $(1 \mathrm{mmol})$ in $10 \mathrm{~mL}$ of hot $\mathrm{EtOH}$ and $10 \mathrm{~mL}$ of $6 \mathrm{~N} \mathrm{HC1}$ were heated under reflux and then $\mathrm{SnCl}_{2} \cdot 2 \mathrm{H}_{2} \mathrm{O}$ was added in portions until the starting material was completely exhausted. The ethanol was decanted; the residue was made alkaline with $\mathrm{KOH}$, then, extracted with EtOAc and washed with water. EtOAc was evaporated slowly and the precipitate re-crystallized from ethanol ${ }^{21}$.

\section{5-Amino-2-phenyl -1-ethyl benzimidazol (8)}

Yield 71\%; m. p.: 199-201 ${ }^{\circ} \mathrm{C}$; IR (KBr, cm $\left.{ }^{-1}\right): 3162(\mathrm{NH}), 2988(\mathrm{CH}), 1620(\mathrm{~N}=\mathrm{C}), 1299$ (C-N stretching), 895 (C-C bonding aromatic); ${ }^{1} \mathrm{HNMR}$ (DMSO-d $\left.{ }_{6}, \delta \mathrm{ppm}\right): 0.73(\mathrm{t}, 3 \mathrm{H}$, $\left.\mathrm{CH}_{3}\right)$, 1.56-1.61 (m, $\left.2 \mathrm{H}, \mathrm{CH}_{2}\right), 2.55\left(3 \mathrm{H}, \mathrm{s}, \mathrm{CH}_{3}\right.$ at $\mathrm{C}-2$ of benzimidazole), $4.22\left(\mathrm{t}, 2 \mathrm{H}, \mathrm{CH}_{2}\right)$, 7.25-7.69 (4H, m, Ar-bbenzimidazole),7.52-7.65 (m, 3H, H-3',4',5'), 7.89-7.96 (m, 2H, H-2',6'), 7.98 (d, 1H, Jo= $8.8 \mathrm{~Hz}, \mathrm{H}-7), 8.11(\mathrm{dd}, 1 \mathrm{H}, \mathrm{Jo}=8.8 \mathrm{~Hz}, \mathrm{Jm}=2 \mathrm{~Hz}, \mathrm{H}-6), 8.46$ $(\mathrm{d}, 1 \mathrm{H}, \mathrm{Jm}=2 \mathrm{~Hz}, \mathrm{H}-4) .13 .05\left(1 \mathrm{H}, \mathrm{s},-\mathrm{NH}-\right.$ benzimidazole). Anal. Calcd. For $\mathrm{C}_{15} \mathrm{H}_{15} \mathrm{~N}_{3}: \mathrm{C}, 75.92$; H, 6.32; N, 17.70; Found: C, 75.85; H, 6.29; N, 17.62; Mass spectra, $m / z=237.30(100 \%)$.

\section{5-Amino-2- (p-Bromophenyl) -1- ethyl benzimidazol (9)}

Yield 75\%, m. p. $130-132{ }^{\circ} \mathrm{C}$; IR $\left(\mathrm{KBr}, \mathrm{cm}^{-1}\right): 3300(\mathrm{NH}), 2923(\mathrm{CH}), 1624(\mathrm{~N}=\mathrm{C}), 1281$ (C-N stretching), 901 (C-C bonding aromatic), 685 (C-Br) ; ${ }^{1} \mathrm{HNMR}$ (DMSO-d ${ }_{6}, \delta \mathrm{ppm}$ ): 0.7 $\left(\mathrm{t}, 3 \mathrm{H}, \mathrm{CH}_{3}\right), 1.62-1.68\left(\mathrm{~m}, 2 \mathrm{H}, \mathrm{CH}_{2}\right), 2.55\left(3 \mathrm{H}, \mathrm{s}, \mathrm{CH}_{3}\right.$ at $\mathrm{C}-2$ of benzimidazole $), 4.12(\mathrm{t}, 2 \mathrm{H}$, $\left.\mathrm{CH}_{2}\right), 4.8\left(\mathrm{~s}, 2 \mathrm{H}, \mathrm{NH}_{2}\right), 6.63(\mathrm{~d}, 1 \mathrm{H}, \mathrm{Jo}=8.4 \mathrm{~Hz}, \mathrm{H}-6), 6.79(\mathrm{~s}, 1 \mathrm{H}, \mathrm{H}-4), 7.23-7.62(4 \mathrm{H}, \mathrm{m}$, Ar-bbenzimidazole) $7.29(\mathrm{~d}, 1 \mathrm{H}, \mathrm{Jo}=8.4 \mathrm{~Hz}, \mathrm{H}-7), 7.36-7.40(\mathrm{~m}, 2 \mathrm{H}, \mathrm{H}-2$ ',6'), 7.74-7.78 (m, 2H, H-3',5'), 13.06(1H,s,-NH-benzimidazole). Anal.Calcd. For $\mathrm{C}_{15} \mathrm{H}_{14} \mathrm{BrN}_{3}: \mathrm{C}, 56.97$; H, 4.46; N, 13.28, Found: C, 56.88; H, 4.41; N, 13.01; Mass spectra, m/z=316.2 (100\%). 


\section{5-Amino- 2- (p- Bromophenyl)- 1- cyclopentyl benzimidazol (10)}

Yield 82\%, m. p. 193-195 ${ }^{\circ} \mathrm{C}$; IR $\left(\mathrm{KBr}, \mathrm{cm}^{-1}\right): 3162(\mathrm{NH}), 2986(\mathrm{CH}), 1654(\mathrm{~N}=\mathrm{C}), 1292.4$ (C-N stretching), 899 (C-C bonding aromatic), 695 (C-Br) ${ }^{1} \mathrm{HNMR}$ (DMSO-d $\left.\mathrm{D}_{6}, \delta \mathrm{ppm}\right)$ 1.63-2.15 (m, 8H, $\left.\mathrm{CH}_{2}\right), 2.55\left(3 \mathrm{H}, \mathrm{s}, \mathrm{CH}_{3}\right.$ at $\mathrm{C}-2$ of benzimidazole $), 4.68-4.77(\mathrm{~m}, 1 \mathrm{H}, \mathrm{CH})$, $4.83\left(\mathrm{~s}, 2 \mathrm{H}, \mathrm{NH}_{2}\right), 6.61(\mathrm{~d}, 1 \mathrm{H}, \mathrm{Jo}=8.8 \mathrm{~Hz}, \mathrm{H}-6), 6.81(\mathrm{~s}, 1 \mathrm{H}, \mathrm{H}-4), 7.28(\mathrm{~d}, 1 \mathrm{H}, \mathrm{Jo}=8.8 \mathrm{~Hz}$, H-7), 7.24-7.61 (4H, m, Ar-bbenzimidazole), 7.36-7.40 (m, 2H, H-3',5'), 7.65-7.69 (m, 2H, H-2',6'); 13.06(1H,s,-NH-Benzimidazole). Anal. Calcd. For $\mathrm{C}_{18} \mathrm{H}_{18} \mathrm{BrN}_{3}$ : C, 60.71; H, 5.05; $\mathrm{N}, 11.79$. Found: $\mathrm{C}, 60.66 ; \mathrm{H}, 5.00 ; \mathrm{N}, 11.66$; Mass spectra, $\mathrm{m} / \mathrm{z}=356.10(100 \%)$.

\section{Antifungal activity assay}

The yeasts Candida albicans, patient isolate Candida glabrata and Candida krusei were grown on sabouraud dextrose broth (Difco); the yeasts were incubated for $48 \mathrm{~h}$ at $25.91{ }^{\circ} \mathrm{C}$. The antifugal activity tests were carried out at pH 7.4 in Sabouraud Dextrose Broth and the 2 -fold dilution was applied. A set of tubes containing only inoculated broth was kept as controls. After incubation for $48 \mathrm{~h}$ at $25.91{ }^{\circ} \mathrm{C}$, the last tube with no yeast growth was recorded to represent minimum inhibitory concentration (MIC), expressed in $\mu \mathrm{g} / \mathrm{mL}$.

\section{Results and Discussion}

Compounds 1 and 2 were prepared from 1-bromo-2,4-dinitrobenzene by reaction with ethyl/cyclopentylamine in DMF according to the literature ${ }^{22}$. The 2-nitro group of compounds 1 and 2 was reduced to 2 -amino (3 and 4) by using $\mathrm{Na}_{2} \mathrm{~S}_{/} \mathrm{NaHCO}_{3}$ in methanol ${ }^{22}$. Condensation of $o$-phenylenediamines (3 and 4) with the $\mathrm{Na}_{2} \mathrm{~S}_{2} \mathrm{O}_{5}$ adduct of appropriate benzaldehydes in $\mathrm{DMF}^{23}$ gave 5-7. Reduction of compounds 5-7 with $\mathrm{SnCl}_{2} \cdot 2 \mathrm{H}_{2} 0$ produced 8-10 (Scheme 1).<smiles>O=[N+]([O-])c1ccc(Br)c([N+](=O)[O-])c1</smiles>

1<smiles>[R12]CC[C@H](C)C(=O)Nc1ccc([N+](=O)[O-])c([N+](=O)[O-])c1</smiles>

$2 \mathrm{a}, \mathbf{R}^{1}=$ Et. $2 \mathrm{~b}, \mathbf{R}^{1}=$ cyclopentyl<smiles>Nc1ccc([N+](=O)[O-])cc1N</smiles>

3a, $\mathbf{R}^{1}=\mathbf{E t}$. $3 \mathbf{b}, \mathbf{R}^{1}=$ cyclopentyl

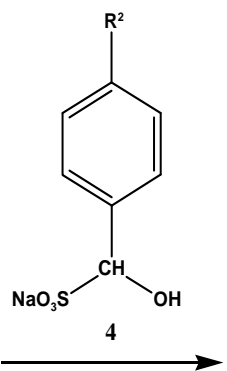

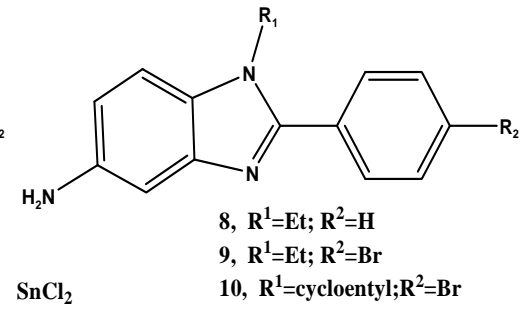

Scheme 1. Preparation rout of the compounds

The in vitro antifungal activity of the compounds was tested by the tube dilution technique ${ }^{24}$. Each of the test compounds and standards miconazole, fluconazole and cotrimoxazole were dissolved in $10 \%$ DMSO, at concentrations of $100 \mu \mathrm{g} / \mathrm{mL}$. Further dilutions of the compounds and standards in the test medium were prepared at the required quantities of $50,25,12.5,6.25,3.125,1.5$ and $0.78 \mu \mathrm{g} / \mathrm{mL}$ concentrations. The final inoculums size was $10^{5} \mathrm{CFU} / \mathrm{mL}$. The MICs were defined as the lowest concentrations of 
the compounds that prevented visible growth. It was determined that the solvent had no antifungal activity against any of the test microorganisms. All the compounds were tested for their in vitro growth inhibitory activity against $C$. albicans, patient isolate C. glabrata and C. krusei (Table 1). Compounds 6, 7, 8 and 9 possessed comparable activity to fluconazole and cotrimoxazole against C. albicans with a MIC of $12.5 \mu \mathrm{g} / \mathrm{mL}$. However none of the compounds was superior to the standards used against any fungi.

Table 1. Antifungal activities of the synthesized compounds

\begin{tabular}{cccc}
\hline Compound & C.albicans & C.glabrata & C.krusei \\
\hline 5 & 25 & 25 & 12.5 \\
6 & 12.5 & 6.25 & 6.25 \\
7 & 12.5 & 25 & 6.25 \\
8 & 12.5 & 25 & 12.5 \\
9 & 12.5 & 12.5 & 12.5 \\
10 & 25 & 25 & 6.25 \\
Fluconazole & 12.5 & 3.125 & 3.125 \\
Miconazole & 6.25 & 3.125 & 1.5 \\
Cotrimoxazole & 12.5 & 3.125 & 3.125 \\
\hline
\end{tabular}

\section{Conclusion}

A series of novel benzimidazole derivatives were successfully synthesized and characterized using IR and ${ }^{1} \mathrm{H}-\mathrm{NMR}$, Mass spectroscopy and elemental analysis. Our studies clearly demonstrate that novel benzimidazole derivatives had significant antifungal activity against different fungi species. As a consequence, we can conclude that newly synthesized benzimidazole derivatives can be used for the development of new fungicide.

\section{Acknowledgment}

The authors gratefully acknowledge for the support from Islamic Azad University, Karaj branch.

\section{References}

1. Utku S, Gokce M, Ozcelik B, Bercin E, Turk J Pharm Sci., 2008, 5(2), 107-116,

2. Kruse L L, Ladd D L, Harrsch P B, McCabe F L, Mong S M, Faucette L and Johnson R, J Med Chem., 1989, 32, 409-417 .

3. Islam I, Skibo E B, Dorr R T and Alberts D S, J Med Chem., 1991, 34, 2954-2961.

4. Habernickel V J, Drugs Made In Germany, 1992, 35, 97.

5. Fukuda T, Saito T, Tajima S, Shimohara K and Ito K, Arzneim.-Forsch./ Drug Res., 1984, 34, 805-810.

6. Nakano H, Inoue $\mathrm{T}$, Kawasaki N, Miyataka H, Matsumoto H, Taguchi T, Inagaki N, Nagai H and Satoh T, Chem Pharm Bull., 1999, 47, 1573-1578.

7. Can-Eke B, Puskullu M O, Buyukbingol E and Ican M, Chem Biol Intract., 1998, 113, 65-77.

8. Kus C, Ayhan-Kilcigil G, B. Can-Eke and Iscan M, Arch Pharm Res., .2004, 27, 156-163.

9. Ayhan-Klcigil G, Ku C, Coban T, Can-Eke B and Lcan M, J Enzyme Inhibition Med Chem., 2004, 19, 129-135.

10. Göker H, Ayhan-Kilcigil G, Tunçbilek M, Kus C, Ertan R, Kendi E, Ozbey S, Fort M, Garcia C and Farre A J, Heterocycl., 1999, 51, 2561-2573. 
11. Abdel-Rahman A E, Mahmoud A M, El-Naggar G M and El-Sherief H A, Pharmazie, 1983, 38(9), 589-590.

12. Soliman F S G, Rida S M, Badawey E A M and Kappe T, Arch Pharm., 1984, 317, 951-958.

13. Coburn R A, Clark M T, Evans R T and Genco R J, J Med Chem., 1987, 30, 205-208.

14. Habib N S, Abdel-Hamid S and El-Hawash M, Farmaco, 1989, 44, 1225-1232.

15. Göker H, Ku C, Boykin D W, Yildiz S and Atlanlar N, Bioorg Med Chem., 2002, 10, 2589-2596.

16. Ozden S, Karata H, Yildiz S and Goker H, Arch Pharm Pharm Med Chem., 2004, 337, 556-562.

17. Ozden S, Atabey D, Yildiz S and Göker H, Bioorg Med Chem., 2005, 13, 1587-1597.

18. Küçflkgflzel I, Küçflkgflzel S G, Rollas S and Kiraz M, Bioorg Med Chem Lett., 2001, 11, 1703-1707.

19. El-Gaby M S A, Micky J A, Taha N M and El-Sharief M A M, J Chin Chem Soc.., 2002, 49, 407-414.

20. Ku C, Göker H, Ayhan G, Ertan R, Antanlar N and Akin A, IIFarmaco, 1996, 51, 413-417.

21. Ayhan Kilcigil G and Altanlar N, Turk J Chem., 2006, 30, 223-228.

22. Willitzer H, Brauniger D, Engelmann D, Krebs D, Ozegowski W and Tonew M, Pharmazie, 1978, 33, 30-38.

23. Ridley H F, Spickett R G W and Timmis G M J, Heterocyclic Chem., 1965, 2, 453-456.

24. Sahm D F and Washington J A, Antibacterial Susceptibility Tests: Dilution Methods, in Manual of Clinical Microbiology, $5^{\text {th }}$ Ed., Eds. Balowes A, Hausler W J, Hermann K L and Shadomy H D, American Society for Microbiology, Washington D C, 1991, 1105-1116. 


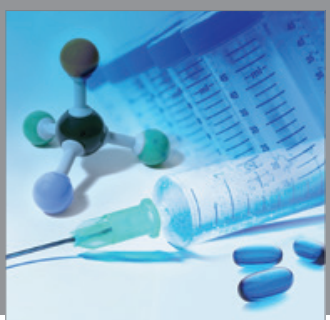

International Journal of

Medicinal Chemistry

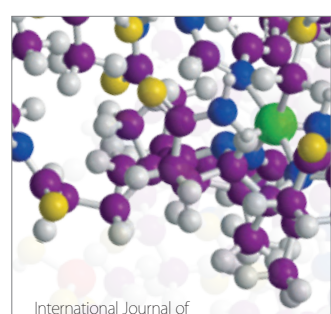

Carbohydrate Chemistry

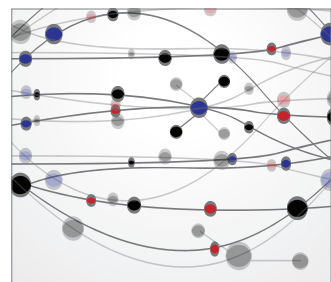

The Scientific World Journal
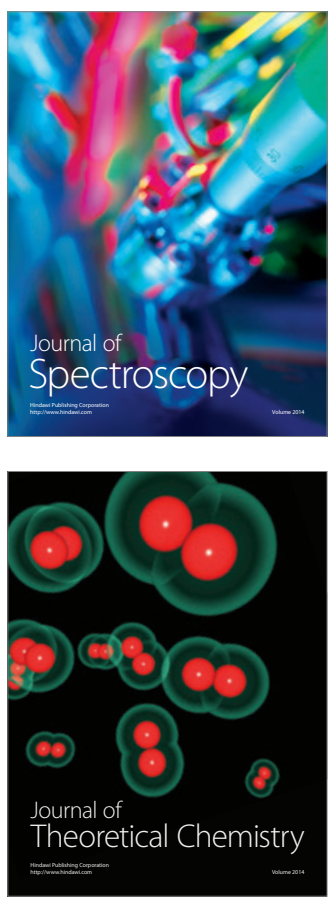
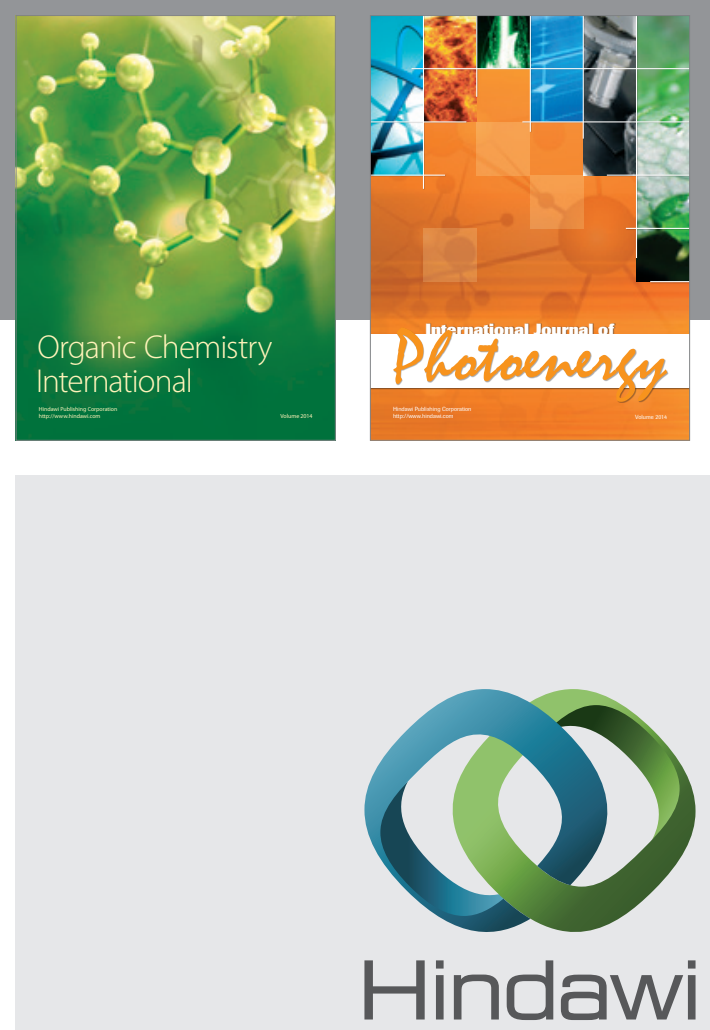

Submit your manuscripts at

http://www.hindawi.com
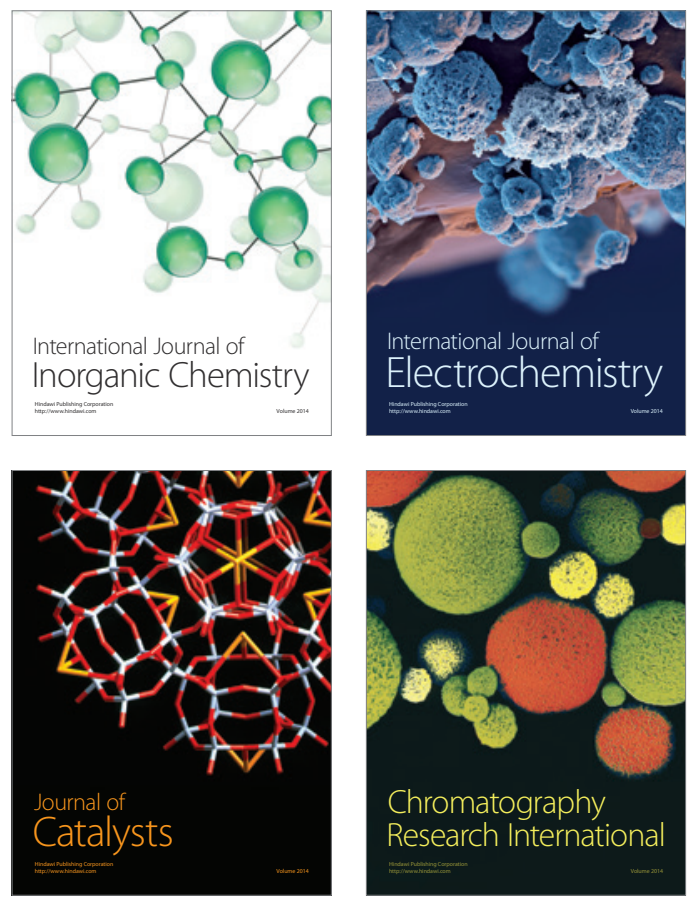
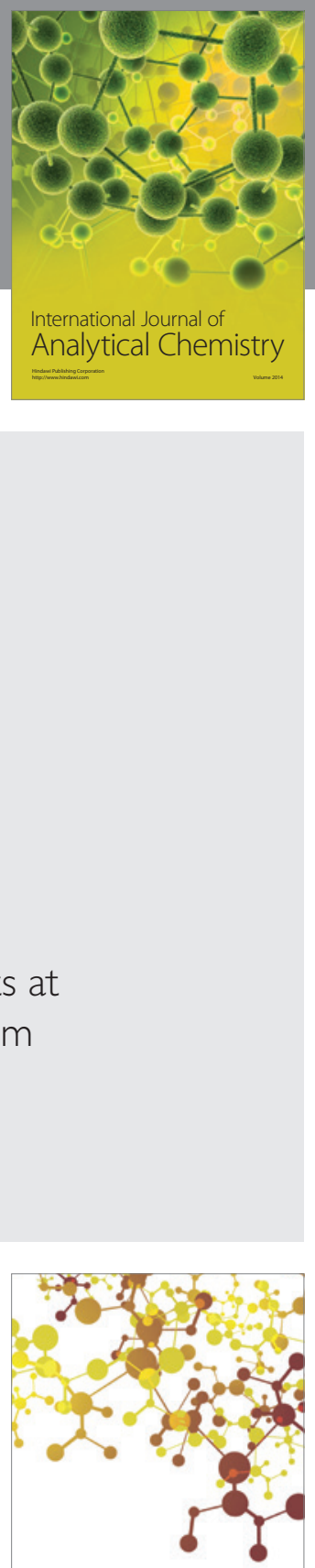

Journal of

Applied Chemistry
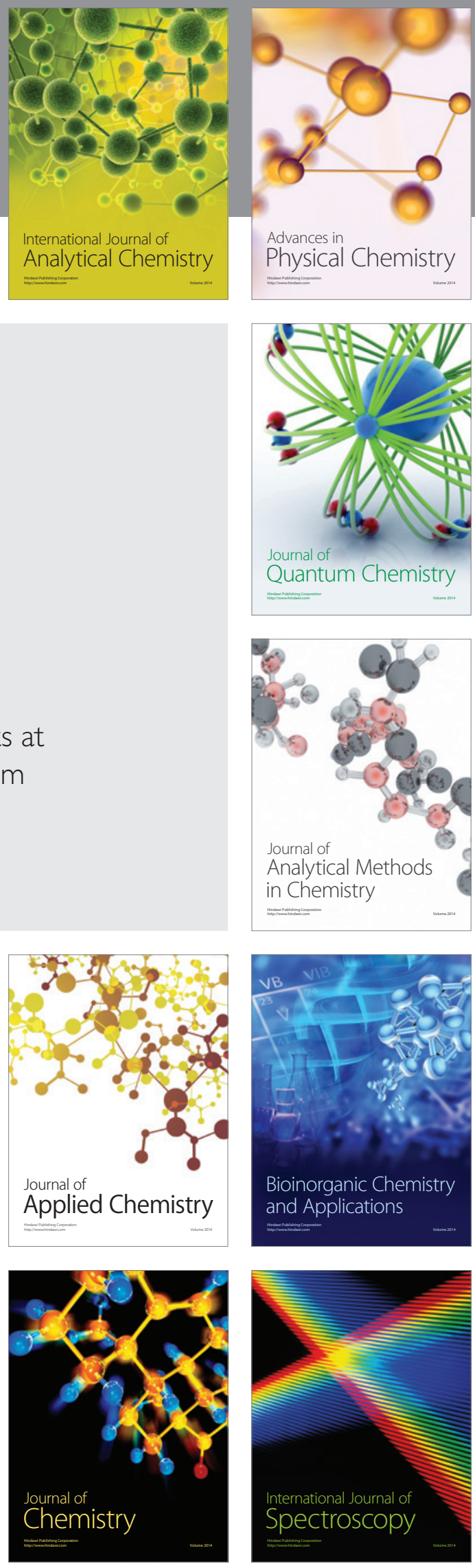